\title{
Desempenho fitométrico do milho sob efeito de doses de trinexapac-ethyl e clomazone
}

\author{
Phytometric performance of corn on the effect of doses of \\ trinexapac-ethyl and clomazone
}

\begin{abstract}
André Prechlak Barbosa ${ }^{1 *}$, Luiz Abilio Ribeiro Alves ${ }^{1}$, Douglas Júnior Bertoncelli ${ }^{1}$, Felipe Favoretto Furlan ${ }^{1}$, Guilherme Augusto Cito Alves ${ }^{1}$, José Henrique Bizzarri Bazzoํㅡ, Claudemir Zucareli ${ }^{1}$
\end{abstract}

Resumo - O incremento produtivo da cultura do milho pode ser maximizado pelo adensamento da lavoura e, para isso, a utilização de herbicidas e reguladores de crescimento, por favorecer a diminuição de porte das plantas, torna-se ferramenta importante. Assim, objetivou-se avaliar doses dos redutores de crescimento trinexapac-ethyl e clomazone sobre o desempenho fitométrico de híbrido simples de milho. O delineamento adotado foi inteiramente casualizado, em esquema fatorial 2 x 5, com quatro repetições. Como primeiro fator considerou-se os redutores de crescimento (trinexapac-ethyl e clomazone) e no segundo foram consideradas as cinco doses de cada redutor $\left(0,50,100,200\right.$ e $400 \mathrm{~g}$ i. a. ha $\left.{ }^{-1}\right)$. Os redutores de crescimento trinexapac-ethyl e clomazone modificam o desempenho fitométrico das plantas de milho. Cerca de $113 \mathrm{~g}$ i.a. ha ${ }^{-1}$ de clomazone proporciona aumento do diâmetro do colmo e da massa fresca. $\mathrm{O}$ herbicida clomazone também pode ser utilizado como redutor de crescimento.

Palavras-chave: herbicida, inibidor de giberelina, regulador de crescimento, Zea mays L.

Abstract - The productive increase of the maize crop can be maximized by crop densification and, for this, the use of herbicides and growth regulators, because it favors the reduction of plant size, becomes an important tool. Thus, the objective of this study was to evaluate the rates of growth reducers on the phytometric performance of maize simple hybrids. The design was completely randomized, in a 2 x 5 factorial scheme, with four replications. Growth factors (trinexapac-ethyl and clomazone) were considered as the first factor and in the second, the five doses of each reducer $\left(0,50,100,200\right.$ and $400 \mathrm{~g}$ i.a. ha $\left.{ }^{-1}\right)$ were considered. The trinexapac-ethyl and clomazone growth modifiers modify the phytometric performance of maize plants. About $113 \mathrm{~g}$ i.a. ha $\mathrm{h}^{-1}$ of clomazone provides an increase in stem diameter and fresh mass. The herbicide clomazone can also be used as a growth-reducing agent.

Keywords: herbicide, gibberellin inhibitor, growth regulator, Zea mays L.

Recebido: Janeiro 26, 2017. Aceito: Janeiro 21, 2018.

${ }^{1}$ Universidade Estadual de Londrina - UEL, Rodovia PR $445 \mathrm{~km}$ 380, CEP 86057-970, Londrina, PR, Brasil.E-mail: andreprechlak@gmail.com; labilioalves@gmail.com; dj_bertoncelli@hotmail.com;

ffavorettofurlan@gmail.com; guilhermecito@hotmail.com; agro.bazzo@gmail.com; claudemircca@uel.br 


\section{Introdução}

O milho (Zea mays L.) é uma cultura de grande relevância econômica e estratégica para a cadeia produtiva de alimentos. Possui elevado potencial produtivo, composição química e valor nutritivo privilegiado, o que o posiciona como um dos cereais mais cultivados e consumidos no mundo. No Brasil, é o cereal mais importante economicamente, principalmente pela geração de empregos na zona urbana e rural além da sua importância para diferentes elos da cadeia do agronegócio (Galvão et al., 2014)

Quanto a eficiência fotossintética, a cultura do milho possui mecanismo bioquímico de concentração do $\mathrm{CO} 2$ nas células da bainha foliar. O incremento produtivo pode ser baseado na otimização da interceptação da radiação solar e conversão em fotoassimilados que formam as estruturas de interesse econômico, sendo essencial para isso que ocorra o máximo aproveitamento da radiação solar, água e nutrientes (Sangoi, Silva, Argenta, Rambo, 2010; Calonego et al., 2011; Taiz e Zeiger, 2013).

Nos últimos anos, o uso de arranjo espacial moderno com maiores densidades populacionais e redução do espaçamento entre linhas de semeadura, associado ao aumento das doses de fertilizante nitrogenados, e condições meteorológicas que estimulam o desempenho vegetativo, têm resultado em limitações, como o crescimento excessivo, o autossombreamento das plantas, além da propensão ao acamamento e quebramento (Argenta, Silva, Sangoi, 2001; Farinelli et al., 2012).

Situações como essas quando não sanadas por genótipos de menor porte que são mais tolerantes ao adensamento e acamamento, exigem a adoção de técnicas alternativas, como o uso de inibidores de crescimento vegetal. Esses são compostos sintéticos, utilizados para reduzir o crescimento indesejável da parte aérea das plantas, sem afetar a produção de grãos (Fernandes, 2009).

O regulador vegetal trinexapac-ethyl interfere na biossíntese do ácido giberélico (Heckman et al.,
2002). Age nas plantas reduzindo a elongação dos entrenós no período vegetativo, interrompe o final da rota metabólica da biossíntese do ácido giberélico, pela inibição da enzima $3 \beta$-hidroxilase, que reduz o teor de giberelina ativa $\left(\mathrm{Ga}_{1}\right)$, pois aumenta seu precursor biossintético imediato $\mathrm{Ga}_{20}$ (Davies, 1987).

Herbicidas também podem ser utilizados como redutores de crescimento, dependendo da dose. Uma molécula possível de ser utilizada é o clomazone que faz parte do grupo das isoxazolinas, pois é absorvido no meristema apical das plantas, pelas raízes e colo, e translocado via xilema até as folhas, onde inibe a formação de geranil fosfato, e retarda a síntese de terpenos (carotenoides, giberelina, etc) (Devine et al., 1993; Rodrigues e Almeida, 1998).

Mediante a falta de trabalhos relacionados a este assunto e a necessidade de ajustar melhor as doses, correlacionada ao melhor redutor de crescimento e seu efeito esperado na cultura, objetivou-se avaliar doses de redutores de crescimento sobre o desempenho fitométrico de híbrido simples de milho.

\section{Material e métodos}

O experimento foi realizado em casa de vegetação, localizada no município de Londrina-PR (2320'32" S e $51^{\circ} 12^{\prime} 32^{\prime \prime} \mathrm{W}$ ), com altitude média de $540 \mathrm{~m}$. As unidades experimentais consistiram de vasos com capacidade de oito litros, preenchidos com Latossolo Vermelho distroférrico (EMBRAPA, 2013). Durante o experimento foi verificado a temperatura e umidade no local, obtendo médias de $18^{\circ} \mathrm{C}$ e $36 \%$ em junho, $19^{\circ}$ e $35 \%$ em julho e $21^{\circ} \mathrm{C}$ e $57 \%$ em agosto.

No dia 1 de junho de 2015, foram colocadas seis sementes por vaso. Quando as plantas atingiram o estádio V3 foi realizado o desbaste, deixando apenas uma planta por vaso (Richie et al.,1993).

A análise química do solo apresentou os seguintes resultados: $\mathrm{pH}\left(\mathrm{CaCl}_{2}\right)=5,50 ; \mathrm{P}=8,55 \mathrm{mg} \mathrm{dm}^{-3}$; $\mathrm{K}=1,60 \mathrm{cmol}_{\mathrm{c}} \mathrm{dm}^{-3} ; \mathrm{Ca}=5,30 \mathrm{cmol}_{\mathrm{c}} \mathrm{dm}^{-3}$; $\mathrm{Mg}=2,20 \mathrm{cmol}_{\mathrm{c}} \mathrm{dm}^{-3} ; \mathrm{Al}=0 \mathrm{cmol}_{\mathrm{c}} \mathrm{dm}^{-3}$; 
$\mathrm{H}+\mathrm{Al}=4,28 \mathrm{cmol}_{c} \mathrm{dm}^{-3} ; \mathrm{CTC}=13,38 \mathrm{cmol}_{\mathrm{c}} \mathrm{dm}^{-3}$ e $\mathrm{V}=68,01 \%$. A adubação de base consistiu da utilização do formulado 08-28-16 na dose de $25 \mathrm{~g}$ por vaso $\left(312,5 \mathrm{~kg} \mathrm{ha}^{-1}\right)$ que disponibilizou $25 \mathrm{~kg}$ de nitrogênio, $87,5 \mathrm{~kg}$ de fósforo e $50 \mathrm{~kg}$ de potássio por hectare.

No estádio V6 foi realizada a adubação de cobertura, utilizando ureia como fonte de nitrogênio, sendo a dose de $70 \mathrm{~kg}$ de $\mathrm{N} \mathrm{ha}^{-1}$. Durante o período experimental foram realizadas irrigações diárias, não sendo necessária a utilização de nenhum produto fitossanitário, visto que a condição de cultivo protegido impediu a ocorrência de pragas e doenças. Todo o manejo realizado está de acordo com as recomendações para a cultura (EMBRAPA, 2012).

Foi utilizado no experimento a cultivar 2B610 PW, híbrido simples transgênico, tolerante a glyphosate, que apresenta resistência ao acamamento. Possui ciclo precoce (860 graus dias). Quanto à altura de plantas, possui porte médio que varia de 1,30 a 2,30 (Dow AgroSciences, 2016).

$\mathrm{O}$ delineamento experimental adotado foi o inteiramente casualizado, em esquema fatorial $2 \times 5$, com quatro repetições. Como primeiro fator considerou-se os redutores de crescimento (trinexapac-ethyl e clomazone) e no segundo foram consideradas as cinco doses de cada redutor $\left(0,50,100,200\right.$ e $400 \mathrm{~g}$ i. a. ha $\left.{ }^{-1}\right)$. Os redutores de crescimento utilizados foram: trinexapac ethyl (Moddus $^{\circledR}, 250 \mathrm{~g} \mathrm{~L}-1$ de i.a., Syngenta) e clomazone (Gamit $360 \mathrm{CS}^{\circledR}, 360 \mathrm{~g} \mathrm{~L}^{-1}$ de i.a., FMC).

A aplicação desses produtos ocorreu no estádio fenológico V6 das plantas de milho, com a utilização de um pulverizador costal pressurizado a $\mathrm{CO}_{2}$, equipado com barra de três metros e pontas tipo leque Teejet XR 8001VS, pressão constante de $200 \mathrm{kPa}$ e taxa de aplicação de $200 \mathrm{~L} \mathrm{ha}^{-1}$.

A avaliação das características fitométricas ocorreu no estádio VT (pendoamento), assim como segue a descrição: a altura das plantas consistiu da determinação em centímetros, por meio da mensuração do comprimento do colmo (da superfície do solo até a base da inflorescência masculina). O número de folhas foi determinado a partir da contagem de folhas da base da planta até a última folha totalmente expandida no ápice da planta. O diâmetro do colmo foi determinado no segundo internódio acima do solo, a partir da base da planta, mensurado com auxílio de paquímetro, e os valores expressos em milímetros.

As massas frescas de folha, e as massas secas de colmo + pendão e total, expressas em gramas, foram determinadas por meio da pesagem em balança analítica, com três casas decimais de precisão após a secagem do material em estufa de circulação forçada de ar, a uma temperatura de $65^{\circ} \mathrm{C}$ por três dias. A porcentagem foliar foi determinada com utilização de um medidor portátil (modelo CI-203 da empresa CID Bio-Science), em todas as folhas da planta.

Os dados foram submetidos a análise de variância e quando constatada diferença, os redutores de crescimento foram comparados pelo teste de $\mathrm{F}$ e as doses de cada redutor por regressão, com significância de $5 \%$ para todos os testes. O software estatístico utilizado foi o SISVAR ${ }^{\circledR}$.

\section{Resultado e discussões}

Não foi constatado efeito de nenhum fator estudado sobre a altura (ALT) e número de folhas (NF). Houve efeito isolado dos reguladores de crescimento (REG) para porcentagem de folha $(\% \mathrm{~F})$. Tratando-se apenas das doses de trinexapac-ethyl e clomazone (DOSE), observou-se efeito para massa seca total (MST). Já para a interação entre os fatores (REG*DOSE) constatou-se efeito para diâmetro do colmo (DC), massa fresca (MF) e massa seca de colmo + pendão (MCP) (Tabela 1).

Para DC, o tratamento trinexapac-ethyl proporcionou melhores respostas às plantas de milho que o clomazone para as doses $200 \mathrm{e}$ $400 \mathrm{~g}$ i. a. ha ${ }^{-1}$, para as demais doses não houve diferença entre os tratamentos (Tabela 2). Resultado semelhante foi obtido por Pricinotto et al. (2015), que trabalhando com milho híbrido, em condição de casa de vegetação, verificaram que o regulador trinexapac-ethyl incrementou o diâmetro do colmo. 
Tabela 1. Resumo da análise de variância para as características avaliadas em função das doses dos redutores de crescimento trinexapac-ethyl e clomazone. Londrina-PR, 2015.

\begin{tabular}{ccccccccc}
\hline FV & GL & ALT & NF & DC & MF & MCP & MST & \% F \\
\hline REG & 1 & $1512,9^{\mathrm{ns}}$ & $0,4^{\mathrm{ns}}$ & $50,63^{* *}$ & $568,74^{* *}$ & $3377,69^{\mathrm{ns}}$ & $1174,51^{\mathrm{ns}}$ & $287,30^{* *}$ \\
ERRO & 27 & 472,54 & 1,53 & 1,55 & 11,03 & 1829,76 & 1872,81 & 26,77 \\
DOSE & 4 & $106,54^{\mathrm{ns}}$ & $0,85^{\mathrm{ns}}$ & $1,6^{\mathrm{ns}}$ & $56,37^{* *}$ & $4676,74^{*}$ & $5474,37^{*}$ & $74,1^{*}$ \\
REG*DOSE & 4 & $209,34^{\mathrm{ns}}$ & $0,15^{\mathrm{ns}}$ & $5,38^{*}$ & $36,08^{*}$ & $494701^{*}$ & $4800,33^{\mathrm{ns}}$ & $34,1^{\mathrm{ns}}$ \\
REP & 3 & $472,97^{\mathrm{ns}}$ & $0,2^{\mathrm{ns}}$ & $0,43^{\mathrm{ns}}$ & $18,64^{\mathrm{ns}}$ & $2265,62^{\mathrm{ns}}$ & $2256,39^{\mathrm{ns}}$ & $42,76^{\mathrm{ns}}$ \\
CV & & 9,79 & 8,66 & 6,71 & 9,65 & 28,74 & 23,61 & 3,13 \\
\hline
\end{tabular}

Notas: FV = Fonte de variação; REG=Regulador de crescimento; DOSE = Doses de redutor de crescimento; REG*DOSE = Interação entre os redutores de crescimento e as doses; REP $=$ Repetição; CV = Coeficiente de variação; GL = Grau de liberdade; ALT = Altura de planta; NF = Número de folhas; DC = Diâmetro de colmo; $\mathrm{MF}=$ Massa fresca; $\mathrm{MCP}=$ Massa seca de colmo + pendão; $\mathrm{MST}=$ Massa seca total; $\% \mathrm{~F}=$ Porcentagem de folhas; $*=$ Significância a $5 \% ; * *=$ Significância a $1 \%$; ${ }^{\text {ns }}=$ Não significativo. Fonte: Autores.

Tabela 2. Características das plantas de milho, em função das doses dos redutores de crescimento trinexapac-ethyl e clomazone. Londrina-PR, 2015.

\begin{tabular}{|c|c|c|c|c|c|c|}
\hline \multirow{3}{*}{ Tratamento } & \multicolumn{6}{|c|}{ Doses de redutor de crescimento } \\
\hline & $\mathbf{0}$ & 50 & 100 & 200 & 400 & Médias \\
\hline & \multicolumn{6}{|c|}{ ALT (cm) } \\
\hline TRI & 209,75 & 212,75 & 225,25 & 211,75 & 220,00 & $215,90 \mathrm{a}$ \\
\hline \multirow[t]{2}{*}{ CLO } & 223,00 & 229,25 & 225,50 & 238,25 & 225,00 & $228,20 \mathrm{a}$ \\
\hline & \multicolumn{6}{|c|}{ NF (unid) } \\
\hline TRI & 14,00 & 14,00 & 14,50 & 14,00 & 14,50 & $14,40 \mathrm{a}$ \\
\hline \multirow[t]{2}{*}{ CLO } & 14,00 & 14,50 & 14,50 & 14,00 & 15,00 & $14,20 \mathrm{a}$ \\
\hline & \multicolumn{6}{|c|}{ DC (mm) } \\
\hline TRI & $20,50 \mathrm{a}$ & $18,00 \mathrm{a}$ & $19,25 \mathrm{a}$ & $19,75 \mathrm{a}$ & $20,00 \mathrm{a}$ & 19,70 \\
\hline \multirow[t]{2}{*}{ CLO } & $17,00 \mathrm{~b}$ & $19,00 \mathrm{a}$ & $19,25 \mathrm{a}$ & $16,50 \mathrm{~b}$ & $16,50 \mathrm{~b}$ & 17,45 \\
\hline & \multicolumn{6}{|c|}{ MF (g) } \\
\hline TRI & $36,49 \mathrm{a}$ & $41,66 \mathrm{a}$ & $40,17 \mathrm{a}$ & $35,76 \mathrm{a}$ & $36,89 \mathrm{a}$ & 38,20 \\
\hline \multirow[t]{2}{*}{ CLO } & $30,96 \mathrm{~b}$ & $30,77 \mathrm{~b}$ & $34,47 \mathrm{~b}$ & $33,13 \mathrm{~b}$ & $23,96 \mathrm{~b}$ & 30,66 \\
\hline & \multicolumn{6}{|c|}{$\mathrm{MCP}(\mathrm{g})$} \\
\hline TRI & $139,64 \mathrm{a}$ & $147,36 \mathrm{a}$ & $135,50 \mathrm{~b}$ & $144,27 \mathrm{a}$ & $131,56 \mathrm{a}$ & 138,67 \\
\hline \multirow[t]{2}{*}{ CLO } & $156,23 \mathrm{a}$ & $185,55 \mathrm{a}$ & $221,50 \mathrm{a}$ & $94,74 \mathrm{a}$ & $132,21 \mathrm{a}$ & 158,04 \\
\hline & \multicolumn{6}{|c|}{ MST (g) } \\
\hline TRI & & 189,02 & 175,67 & 180,04 & 168,46 & $177,86 \mathrm{a}$ \\
\hline \multirow[t]{2}{*}{ CLO } & & 216,31 & 255,97 & 127,88 & 159,15 & $188,70 \mathrm{a}$ \\
\hline & \multicolumn{6}{|c|}{$\% \mathbf{F}$} \\
\hline TRI & & 22,60 & 23,05 & 24,88 & 22,34 & $22,74 \mathrm{a}$ \\
\hline CLO & & 15,08 & 13,60 & 26,10 & 15,51 & $17,38 \mathrm{~b}$ \\
\hline
\end{tabular}

Notas: TRI $=$ trinexapac-ethyl; CLO = clomazone; $\mathrm{ALT}=$ Altura de planta; $\mathrm{NF}=$ Número de folhas; $\mathrm{DC}=$ Diâmetro de colmo; $\mathrm{MF}=$ Massa fresca; $\mathrm{MCP}=$ Massa seca de colmo + pendão; $\mathrm{MST}=$ Massa seca total; $\% \mathrm{~F}=$ Porcentagem de folhas. $\mathrm{cm}=$ centímetros; unid $=$ unidades; $\mathrm{mm}=$ milímetros; $\mathrm{g}=$ gramas; $\%=$ porcentagem. Valores seguidos de mesma letra na coluna não diferem pelo teste $\mathrm{F}$ a $5 \%$ de probabilidade. Fonte: Autores.

Houve ajuste de curva de regressão apenas para o clomazone, proporcionando máximo diâmetro até a dose de $113,25 \mathrm{~g}$ i. a. ha ${ }^{-1}$ e reduções na variável com o incremento das doses (Figura 1e).

Essa resposta do redutor se deve ao encurtamento dos entrenós do colmo do milho, devido a redução do teor de ácido giberélico nos meristemas intercalares, isso porque o trinexapac-ethyl atua inibindo a biossíntese de giberelina, em que a rota é obstruída na passagem de $\mathrm{Ga}_{20}$, uma forma inativa, para $\mathrm{Ga}_{3}$ que é uma forma ativa do hormônio (Rademacher, 2015). 

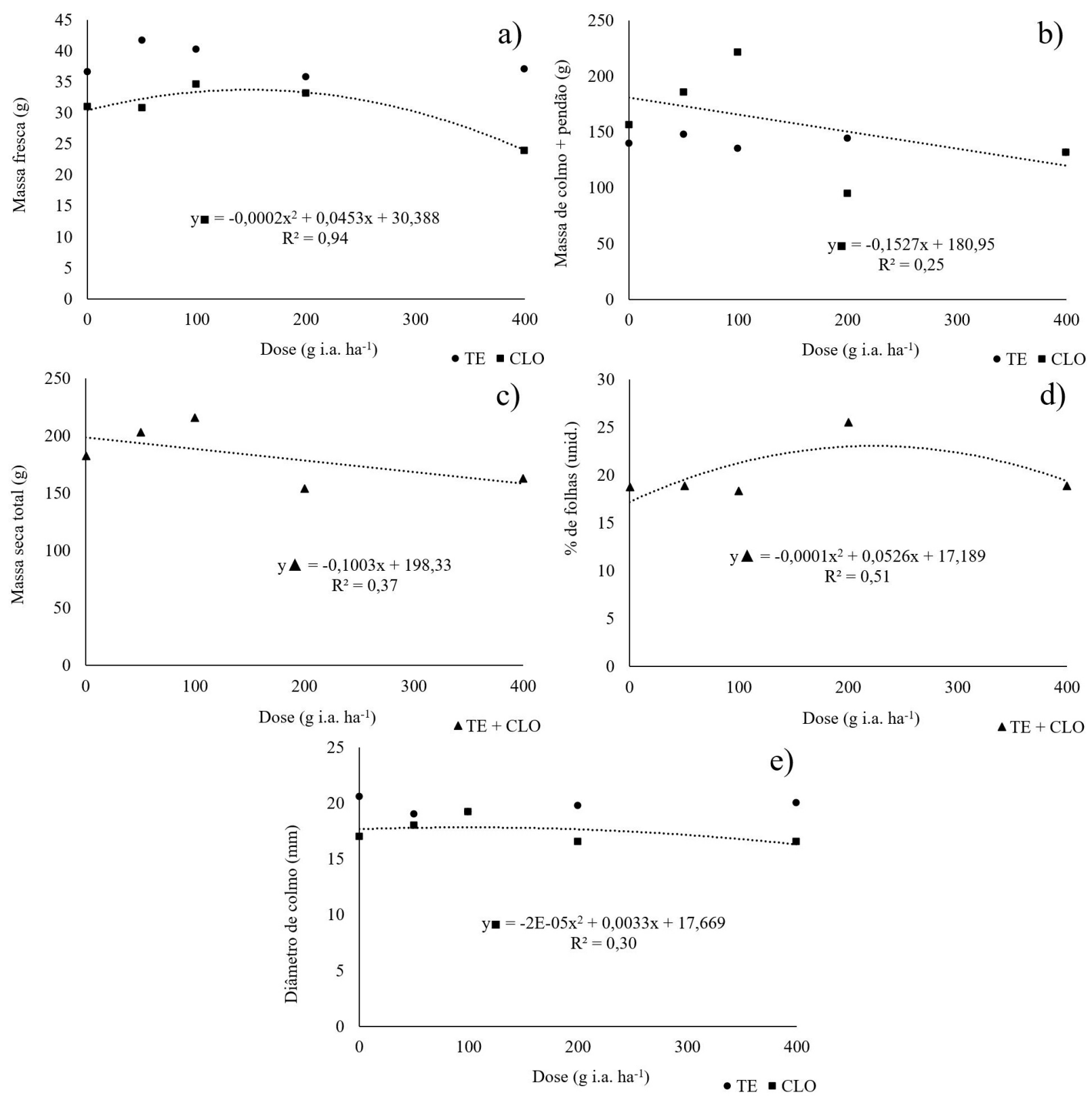

Figura 1. Características fitométricas do milho em resposta a doses de trinexapac-ethyl e clomazone. Londrina-PR, 2015.

Para MST, o clomazone proporcionou maior acúmulo para a dose de $100 \mathrm{~g}$ i. a. ha ${ }^{-1}$ que o trinexapac-ethyl, já nas demais doses não houve diferença entre os reguladores de crescimento (Tabela 2).

Costa et al. (2009) ao trabalhar com quatro espécies de gramas e o redutor de crescimento trinexapac-ethyl, obtiveram resposta diferente da encontrada no presente trabalho, em que houve redução da massa fresca das plantas conforme incrementou-se a dose do redutor.

Dinalli et al. (2015) e Marchi et al. (2013) ao testarem os reguladores trinexapac-ethyl, prohexadione-calcium, bispyribac-sodium e os herbicidas glyphosate, imazaquin, imazethapyr, e metsulfuron-methyl como redutores de crescimento 
em gramados, observaram distintas respostas entre eles, com maior efetividade dos reguladores em reduzir a massa seca e o porte das plantas, semelhante ao verificado para o trinexapac-ethyl no presente estudo.

A variável MF apresentou efeito da interação, porém, houve ajuste apenas para o tratamento com clomazone, o qual apresentou adequação quadrática com ponto de máximo acúmulo de matéria fresca na dose de 113,3 g. i. a. ha ${ }^{-1}$, como mostra a Figura 1a. Isto está relacionado com o modo de ação do clomazone, ou seja, sua ação como inibidor da síntese de carotenoides, em que o mesmo interfere na rota do ácido malônico, e por consequência na síntese dos terpenos (rota independente do ácido mevalônico), nos cloroplastos, e podem, portanto, diminuir a síntese de giberelinas e reduzir o crescimento vegetal (Kerbauy, 2004).

Para MCP, o efeito apresentado resultou em interação, porém, houve ajuste apenas para o tratamento com clomazone, o qual apresentou adequação linear decrescente conforme houve o aumento das doses (Figura 1b). Este efeito também está relacionado com o modo de ação do clomazone citado acima.

Para a \% F, assim como para MST, o efeito foi isolado para as doses dos redutores, em que esta apresentou a maior porcentagem de folha na dose de 262,5 g. i. a. ha-1, como mostra a Figura 1d. Esse comportamento relaciona-se diretamente com a redução de massa e porte das plantas, principalmente do colmo que é mais afetado pela inibição da síntese de giberelinas, fazendo com que até a dose supramencionada, a percentagem de folhas das plantas fosse maior.

Resultado semelhante foi observado por Espindula et al. (2010) que, ao trabalhar com redutores de crescimento na cultura do trigo, obtiveram a redução do crescimento do colmo das plantas e por consequência incremento na percentagem de folhas da cultura.

\section{Conclusões}

Os redutores de crescimento trinexapac-ethyl e clomazone modificam o desempenho fitométrico das plantas de milho.

A dose de 113 g i.a. ha ${ }^{-1}$ de clomazone proporciona aumento do diâmetro do colmo e da massa fresca.

O herbicida clomazone também pode ser utilizado como redutor de crescimento.

\section{Referências}

Argenta, G.; Silva, P.R.F.; Sangoi, L. Arranjo de plantas em milho: análise do estado-da-arte. Ciência Rural, v.31, n.6, p.1075-1084, 2001.

Calonego, J.C.; Poleto, L.C.; Domingues, F.N.; Tiritan, C.S. Produtividade e crescimento de milho em diferentes arranjos de plantas. Agrarian, v.4, n.12, p.84-90, 2011.

Costa, N.V.; Martins, D., Rodella, R.A., Rodrigues, A.C.P., Cardoso, L.A. Características morfológicas de gramas em resposta à aplicação de trinexapacethyl. Planta Daninha, v.27, n.1, p.113-122, 2009.

Davies, P.J. The plant hormones: their nature, occourrence, and functions. In. Plant hormones and their role in plant growth and development. Nethrlands: Kluwer Academic, s/v, s/n, p. 1-23, 1987.

Devine, M.D.; Duke, S.O.; Fedtke, C. Safeners for herbicides. In: Devine, M.D.; Duke, S.O.; Fedtke, C. (Ed.). Physiology of herbicide action. Englewood Cliffs: NJ. Prentice Hall, 1993. p.376-387.

Dinalli, R.P.; Buzetti, S.; Gazola, R.N.; Catilho, R.M.; Celestrino, T.S.; Dupas, E.; et al. Application of herbicides as growth regulators of emerald Zoysia grass fertilized with Nitrogen. Semina: Ciências Agrarias, v.36, n. 3, p.1875-1894, 2015. Edição Suplementar.

Dow AgroSciences. Guia de sementes 2016. Disponível em: <http://msdssearch. 
dow.com/PublishedLiteratureDAS/ dh_0965/0901b803809657ef.pdf?filepath=br/ pdfs/noreg/013-05181.pdf\&fromPage $=$ GetDoc $>$. Acesso em: 22 nov. 2017.

EMPRESA BRASILEIRA DE PESQUISA AGROPECUÁRIA - EMBRAPA. Sistema Brasileiro de Classificação de Solos. 3.ed. Brasília: EMBRAPA, 2013. 353p.

EMPRESA BRASILEIRA DE PESQUISA AGROPECUÁRIA - EMBRAPA. Sistemas de produção: cultura do milho. 8.ed. 2012. Disponível em: https://www.spo.cnptia.embrapa. br/conteudo?p_p_id=conteudoportlet_WAR_ sistemasdeproducaolf6_1ga1ceportlet\&p_p lifecycle $=0 \& p \_p \_s t a t e=$ normal\&p_p $\operatorname{mode}=\mathrm{view} \& \mathrm{p} \_$p_col_id=column3\&p_p_col_count $=1 \&$ p_r_p_-76293187 sistemaProducaold $=3821 \&$ p_r_p_-996514994_ topicoId=3715. Acesso em: 20 nov. 2017.

Espindula, M.C.; Rocha, V.S.; Souza, L.T.; Souza e José, M.A.; Grossi, A.S. Efeitos de reguladores de crescimento na elongação do colmo de trigo. Acta Scientiarum. Agronomy, v.32, n.1, p.109116, 2010.

Farinelli, R.; Penariol, F.G.; Fornasieri Filho, D. Características agronômicas e produtividade de cultivares de milho em diferentes espaçamentos entre linhas e densidades populacionais. Científica, v.40, n.1, p.21-27, 2012.

Fernandes, A.C. População de plantas e regulador de crescimento afetando a produtividade de cultivares de trigo. 2009. 100 f. Dissertação (Mestrado em Agronomia) - Universidade Estadual de Ponta Grossa, Ponta Grossa, 2009.

Galvão, J.C.C.; Miranda, G.V.; Trogello, E.; Fritsche-Neto, R. Sete décadas de evolução do sistema produtivo da cultura do milho. Revista Ceres, v.61, p.819-828, 2014.

Heckman, N.L.; Elthon, T.E.; Horst, G.L.; Gaussoin, R.E. Influence of trinexapac-ethyl on respiration of isolated wheat mitochondria. Crop Science, v.42, n.2, p.423-427, 2002.

Kerbauy, G.B. Fisiologia vegetal. Rio de Janeiro: Guanabara Koogan, 2004. 453p.

Marchi, S.R.; Martins, D.; Costa, N.V.; Silva, J.R.V. Efeito de reguladores vegetais sobre o crescimento e o florescimento de grama-esmeralda. Planta Daninha, v.31, n.3, p.695-703, 2013.

Pricinotto, L.F.; Zucareli, C.; Batista e Fonseca, I. C.; Oliveira, M.A.; Ferreira, A.S.; Spolaor, L.T. Trinexapac-ethyl in the vegetative and reproductive performance of corn. African Journal of Agricultural Research, v.10, n.14, p.1735-1742, 2015.

Rademacher, W. Plant growth regulators: backgrounds and uses in plant production. Journal of Plant Growth Regulation, v.34, n.4, p.845-872, 2015.

Ritchie, S. W.; Hanway, J. J.; Benson, G. O.; Herman, J. C. How a corn plant develops. Iowa: Iowa State University of Science and Technology, Cooperative Extension Service, 1993.

Rodrigues, B.N.; Almeida, F.S. Clomazone: guia de herbicidas. 4.ed. Londrina: IAPAR, 1998. p.137-142.

Sangoi, L.; Silva, P.R.F.; Argenta, G.; Rambo, L. Ecofisiologia da cultura do milho para altos rendimentos. Lages: Editora Graphel, 2010. p.87.

Taiz, L.; Zeiger, E. Fisiologia vegetal. 5.ed. Porto Alegre: Artmed, 2013. p.918. 